\title{
Preparation of Zoledronate liposome and its impact on apoptosis of Kupffer cells in rat liver ${ }^{1}$
}

Qiao-Hong Zhao', Xi-Shan Zhang", Kun Wu'"', Jie Zhang'"', Tian-Fang Xia'", Jian Chen"', Zhen-Shen Qin'v, Li-Qun PangIV

'Bachelor of Medical Science, Department of Nursing, Jiangsu College of Nursing, Huai'an, China. Conception of the study, acquisition and interpretation of data.

"Bachelor of Medical Science, Department of General Surgery, Lian'shui County People's Hospital, Lian'shui, China. Analysis and interpretation of data.

I'Fellow Master degree, Postgraduate Program in Surgical Science, Department of General Surgery, The Affiliated Huaian No.1 People's Hospital of Nanjing Medical University, Huai'an, China. Immunohistochemical and flow cytometry analysis. IVPhD, Associate Professor, Department of General Surgery, The Affiliated Huaian No.1 People's Hospital of Nanjing Medical University, Huai'an, China. Conception and design of the study, critical revision.

\begin{abstract}
Purpose: To establish a method for the preparation of zoledronate liposome and to observe its effect on inducing the apoptosis of rat liver Kupffer cells.

Methods: Zoledronate was encapsulated in liposomes, and then the entrapment rate was detected on a spectrophotometer. The prepared Zoledronate liposome $(0.01 \mathrm{mg} / \mathrm{mL})$ was injected into the tail vein of SD rats. Three days later, the number of Kupffer cells (CD68 positive) in rat liver tissue was detected by immunohistochemistry. Flow cytometry was used to detect the apoptosis rate of the isolated liver Kupffer cell cultured in vitro.

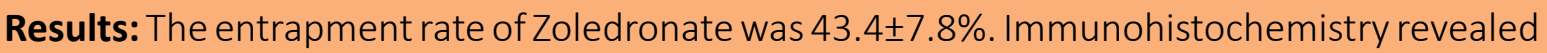
that the number of Kupffer cells was $19.3 \pm 2.1$ in PBS group and $5.5 \pm 1.7$ in Zoledronate liposome group, with a significant difference $(P<0.05)$. The apoptosis rate of Kupffer cells was $4.1 \pm 0.8 \%$ in PBS group, while it was $9 \pm 2.2 \%$ and $23.3 \pm 5.9 \%$ in Zoledronate liposomes groups with different concentrations of Zoledronate liposome $(P<0.05)$.

Conclusions: Zoledronate liposomes can effectively induce the apoptosis of Kupffer cells in vivo and in vitro, and the apoptosis rate is related to the concentration of Zoledronate liposome. To establish a rat liver Kupffer cell apoptosis model can provide a new means for further study on Kupffer cell function.

Key words: Kupffer Cells. Zoledronate. Liposomes. Liver. Rats.
\end{abstract}




\section{Introduction}

The first closed bilayer phospholipid systems, called liposomes, is described in 1965 and soon were proposed as drug delivery systems that can reduce the toxic effects of drugs and mainly be phagocytized by the mononuclear macrophage system of liver and spleen in the body ${ }^{1}$. The pioneering work of countless liposome researchers over almost 50 years led to the development of important technical advances such as long-circulating (PEGylated) liposomes, triggered release liposomes, liposomes containing nucleic acid polymers, ligand-targeted liposomes and liposomes containing combinations of drugs ${ }^{2}$.

Zoledronate (ZOD), a nitro diphosphonate, was the third generation of bisphosphonates, which was more potent and safe to induce osteoclast apoptosis to cure bone tumors in clinics, while the mechanism is the formation of toxic analogues of ATP in osteoclasts (macrophages residing in the bone) after they phagocytize the bisphosphonate bound to hydroxyapatite crystals leads to the intracellular energy metabolism disorder and causes osteoclast apoptosis, thereby reducing osteoclast absorption of bone ${ }^{3,4}$. In addition to inhibition of cell multiplication and initiation of apoptosis in cultured cancer cells ${ }^{5}$, ZOD was widely used in giant cell tumor of bone, multiple myeloma, metastatic bone cancer and not only suppress the progression to bone metastatic lesions but also prevented growth of primary hepatocellular carcinoma $a^{6-8}$. However, because of the short half-life and rapid uptake and accumulation within bone, its use as antitumor agent for extra-skeletal malignancies was limited ${ }^{9}$. ZOD-containing selfassembly PEGylated nanoparticles (NPs) based on ZOD complexes with calcium phosphate NPs (CaPZ NPs) and cationic liposomes was developed to meet the clinical needs ${ }^{10}$ and it has been found that with the injection of ZOD into mice bearing tumor, the vascular structure in the tumor was induced changes, which then was enhanced the antitumor efficacy by the use of liposomal ${ }^{11}$.

For this reason, Zoledronate was used in this study to prepare liposome-encapsulated Zoledronate for intravenous injection to selectively induce apoptosis of Kupffer cells (KCS) in rat liver, which laid the foundation for further study of its function.

\section{- Methods}

All experimental operations were approved by the Ethics Committee of Jiangsu University. This study was carried out in strict accordance with the recommendations in the Guide for the Care and Use of Laboratory Animals of the National Institutes of Health. The animal use protocol has been reviewed and approved by the Institutional Animal Care and Use Committee (IACUC) of Nanjing Medical University. The approval number was KY-P-2016-007-01.

Specific pathogen free (SPF) male Sprague Dawley (SD) rats (license number: SCXK: (Su) 2009-0002), aged 10-12 weeks and weighing 180-280 g, were provided by the Animal Center of Jiangsu University, China. Animals were fed in cages with controlled temperature and humidity at the Animal Center of Jiangsu University, China. Animals were free for foods and drinking.

\section{Preparation of liposomes}

We used the methods reported by Van Rooijen et $a . .^{12}$ and made some improvement. In brief, $8 \mathrm{mg}$ cholesterol was dissolved in 10 $\mathrm{mL}$ chloroform. Then $0.86 \mathrm{~mL}$ of the stock solution of phosphatidylcholine was added to remove the chloroform, followed by elution with $0.3 \mathrm{M}$ phosphate buffered Zoledronate (20 $\mathrm{mg}$ Zoledronate dissolved in $10 \mathrm{~mL}$ phosphate buffer) to diffuse the phospholipid membrane, forming a milky suspension. After stored in Nitrogen for $2 \mathrm{~h}$, the suspension was shocked 
for $3 \mathrm{~min}$ in a water bath sonicator and then stored at room temperature for $2 \mathrm{~h}$ to expand liposomes. Then liposomes were washed 2-3 times in sterile phosphate buffer saline (PBS) to remove the uncoated Zoledronate. Finally, the precipitated liposomes were suspended in $4 \mathrm{~mL}$ PBS to form a milky colloidal solution.

\section{Determination of entrapment rate}

Drug solution at $0.1 \mathrm{mg} / \mathrm{mL}$ was diluted into $0.01 \mathrm{mg} / \mathrm{mL}$. Appropriate amount of diluted drug solution, empty liposome, Zoledronate lipidsome were scanned in full wavelength including UV and visible light to determine its maximum absorption wavelength. PBS solution served as a blank control. Zoledronate was separated by dissolving the liposomes in chloroform/methanol mixture (concentration ratio 9:1) since with nitric acid zoledronate would form a colored complex with copper ions. The concentration of Zoledronate was determined by ultraviolet spectrophotometer at $240 \mathrm{~nm}$. The results showed that the encapsulation efficiency of Zoledronate was $(43.4+7.8) \%$.

\section{Drug loading of zoledronate liposome}

$4 \mathrm{~mL}$ Zoledronate liposome loading $=$ entrapment ratextotal dosage $=(43.4 \pm 7.8)$ $\% \times 20(\mathrm{mg})=8.68(\mathrm{mg})$, so the loading dose of Zoledronate liposome is $8.68 / 4=2.17(\mathrm{mg} / \mathrm{ml})$.

\section{Animal grouping and treatment}

Food fasting for $12 \mathrm{~h}$ to avoid reflex and aspiration during anesthesia for better recover after operation and liquid fasting for $6 \mathrm{~h}$ to all of rats before treatment. Twenty SD male rats were randomly divided into two groups: control group (Group A, treated with PBS by tail vein injection) and Zoledronate liposome group (Group B, treated with Zoledronate liposome $(0.01 \mathrm{mg} / \mathrm{mL})$ by tail vein injection). Three days later, the rats were sacrificed and the liver was collected for paraffin embedding.

Isolation of rat liver Kupffer cells

Rat liver tissue was minced and disgested with collagenase in a water bath at $37^{\circ} \mathrm{C}$ for $20 \mathrm{~min}$. then the tissue mince was isolated by density gradient centrifugation. KCs were purified by adherent method.

Immunohistochemical detection (IHC)

Liver sections underwent conventional dewaxing and hydration, and then applied for IHC using streptavidin-perosidase (SP) method. Sections were incubated with $3 \% \mathrm{H}_{2} \mathrm{O}_{2}$ for 5 min, and then blocked by $10 \%$ normal goat serum for $10 \mathrm{~min}$ at room temperature, followed by incubation with mouse anti-rat CD68 antibody at $37^{\circ} \mathrm{C}$ for $2 \mathrm{~h}$. Subsequently, sections were incubated with biotin-labeled secondary antibody (1:200 goat anti-mouse IgG) for 30 min, followed by incubated with streptavidin labeled with streptozotocin (1:200) for $30 \mathrm{~min}$. Then the sections were developed with DAB and counterstained with Hematoxylin. After mounting, sections were observed under a light microscope. Cytoplasm showed brown or brown particles were defined as positive. The $\mathrm{IHC}$ results were quantitatively analyzed using an image analysis software.

\section{Flow cytometry}

The rat liver cells were made into cell suspension and isolated by gradient centrifugation. After adjusting the cell concentration, cells were inoculated in 24well plates ( $1 \mathrm{~mL}$ per well) and incubated in an incubator with $5 \% \mathrm{CO}_{2}$ at $37^{\circ} \mathrm{C}$ for $1 \mathrm{~h}$. Then the plates were taken out. All non-adherent cells were washed off, and the left adherent cells were Kupffer cells. $1 \times 10^{6}$ Kupffer cells were divided into three groups: group $A$ : treated with PBS; group B: treated with Zoledronate liposomes $(0.01 \mathrm{mg} / \mathrm{mL})$; group $\mathrm{C}$ : treated with 
Zoledronate liposomes $(0.03 \mathrm{mg} / \mathrm{mL})$. After cultured at $37^{\circ} \mathrm{C}$ and $5 \% \mathrm{CO}_{2}$ for $2 \mathrm{~h}$, cells were metered volume with $100 \mu \mathrm{L}$ staining buffer, and incubated with $1 \mu \mathrm{L}$ FITC-labeled antiCD11C antibody at $4^{\circ} \mathrm{C}$ for 20-30 min in dark. Then the cells were washed with PBS and resuspended in $100 \mu \mathrm{L}$ PBS and detected on a flow cytometer.

\section{Statistical analysis}

Statistical analysis was performed using SPSS v17.0 package. Data were expressed as mean \pm standard deviation (SD). Comparisons between the two groups were carried out using $t$ test. $\mathrm{P}<0.05$ was considered as significant different.

\section{- Results}

\section{Entrapment rate of zoledronate}

Zoledronate can react with copper ions and form colored compound in the presence of nitric acid. For this reason, Zoledronate liposome was dissolved in a chloroform/ methanol mixed solution (concentration ratio of 9:1) to separate Zoledronate, so that its concentration could be detected by ultraviolet spectrophotometer at $240 \mathrm{~nm}$. The results showed that the entrapment rate (\%) of Zoledronate was $43.4 \pm 7.8$.

\section{Number of Kupffer cells}

Immunohistochemical staining showed Kupffer cells settled in the hepatic sinusoid with irregular shape. Most of the cell body intruded into or completely mobilized into the hepatic sinusoid. They extended into the perisinusoidal space and directly contacted with hepatocytes (Figure 1A). After treated with PBS or Zoledronate liposomes, the numbers of Kupffer cells (positive for CD68) were $(19.3 \pm 2.1)$ in PBS group and $(5.5 \pm 1.7)$ in Zoledronate liposome group, with a significant difference $(P<0.05$, Figure $1 B)$.

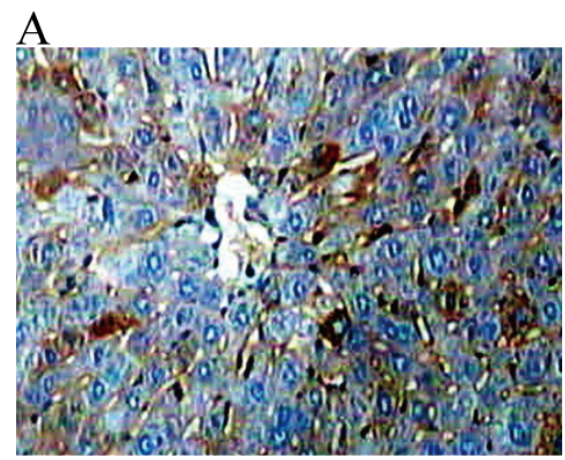

B

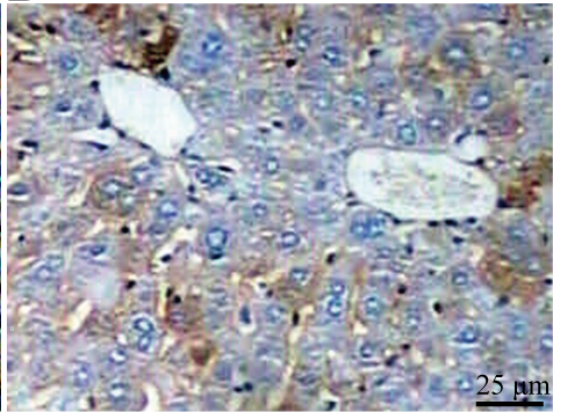

Figure 1 - CD68 ${ }^{+}$Kupffer cells in liver tissue of the two groups determined by IHC (×400). A: PBS group; B: Zoledronate liposome group.

Apoptosis rate of $K C S$

The apoptosis rates of KCs were $4.1 \pm 0.8 \%$ in PBS group and $9.0 \pm 2.2 \%$ and
$23.3 \pm 5.9 \%$ in Zoledronate liposome group with different concentrations, showing significant differences $(P<0.05$, Figure 2$)$. 


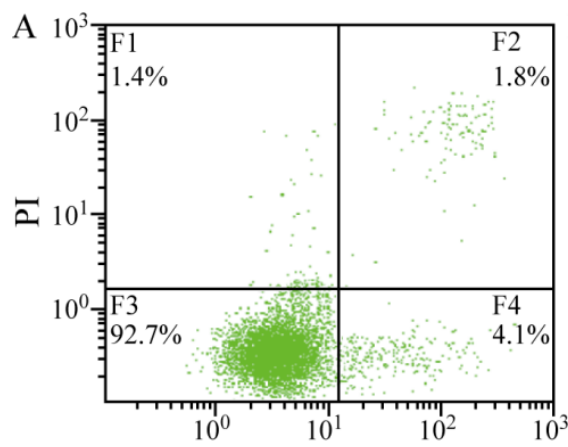

FITC

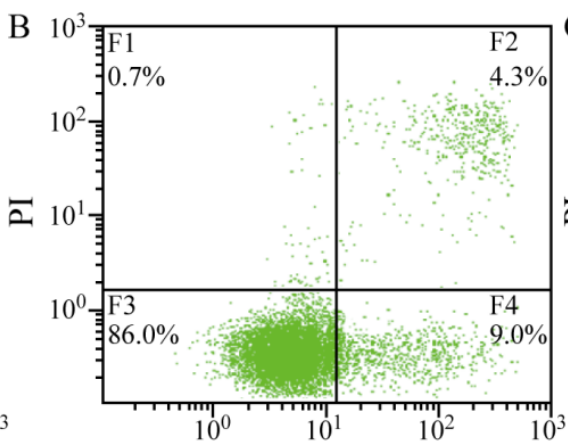

FITC

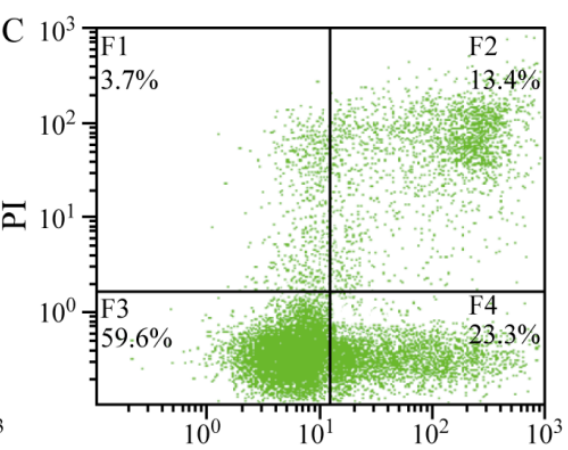

FITC

Figure 2 - The apoptosis rates of KCs determined by flow cytometry. A: PBS group; B: Zoledronate liposome group $(0.01 \mathrm{mg} / \mathrm{mL})$; C: Zoledronate liposome group $(0.03 \mathrm{mg} / \mathrm{mL})$.

\section{- Discussion}

Kupffer cells (KCs) locate in hepatic sinusoids and attach to the endothelial cells. They extend into the sinus space through the fenestra. The amount of KCs accounts for 35\% of the non-parenchymal cells in the liver and $80 \%-90 \%$ of the total monocytes-macrophages system $^{13}$. Studies have shown that KCs have strong phagocytosis which can be enhanced or inhibited by a variety of drugs. Otogawa et $a . .^{14}$ found that the expression of dead ligands and cytokines in KCs is increased after they phagocytize the apoptotic bodies, which aggravates the inflammation and fibrosis of liver ${ }^{15}$. KCs can secrete and synthesize a variety of bioactive substances such as tumor necrosis factor $\alpha$ (TNF- $\alpha)$, interleukin, platelet-activating factor (PAF), reactive oxygen species (OFR), nitric oxide (NO) and so on. They regulate the bodyss immune response through inducing the proliferation of lymphocytes, secreting cytokines and chemokines, expressiing certain molecules and other pathways ${ }^{16}$. Recent studies have shown that PK2/Bv8 expression of KCs is decreased in liver cancer as compared with that in normal liver ${ }^{17}$. KCs are involved in the hepatic metastasis from gastrointestinal malignant tumors promoted by carcinoembryonic antigen (CEA) and the carcinogenesis of peroxisome proliferator.
Meanwhile, KCs also participate in the carcinogenesis of hepatocarcinogen through producing inflammatory cytokines such as superoxide. In addition, KCs are also involved in tumor immunity through phagocytosis, antibody-dependent cell-mediated cytotoxicity, antigen presentation and release of cytokines.

It has been found that the expression of CD40/CD40L of KCs plays a role in chronic rejection following liver transplantation. KCs also exacerbate self-apoptosis by secreting NO, leading to acute rejection in liver transplantation. At the same time, KCs induce T cell apoptosis and differentiation through the Fas-FasL apoptotic pathway, playing a role in the tolerance of liver transplantation ${ }^{18,19}$.

In the early stage of transplantation and reperfusion, cytokines such as TNF- $\alpha$, IL-1 and IL-6 released by the activated Kupffer cells and a large number of reactive oxygen species (ROS) cause direct damage to hepatocytes, while the late injury is the serious injury on hepatocytes caused by complicated inflammatory cascade induced by neutrophil infiltration, release of a large number of oxygen free radicals and protease and microcirculation disturbance of the grafted liver ${ }^{20-22}$. The activation of $\mathrm{KCS}$ and the over-expression of inflammatory factors, such as TNF- $\alpha$ are main initiating factors causing the persistent imbalance of proinflammatory/ 
anti-inflammatory self-stabilizing mechanism in vivo and leading to the inflammatory cascade reaction, which further leads to the pathological process of IRI and grafted liver organ failure. Effective regulation of the function of KCs may become an effective way to prevent and treat the IRI in transplanted liver.

At present, the inhibition and regulation of KC function has become one of the hotspots in the researches of protective measure from IRI in transplanted liver. In the past five years, 40 researches have been retrieved on PubMed by the MESH "liver transplantation AND Kupffer word cell AND ischemia reperfusion injury". Most of them used taurine, carbon monoxide and hemeoxygenase 1 (HO-1) to inhibit the secretion and phagocytosis of $\mathrm{KCs}$, or used gadolinium chloride $(\mathrm{GdCl} 3)$ to induce the apoptosis of $\mathrm{KCs}$, in order to reduce the hepatic ischemia-reperfusion injury ${ }^{23-26}$. Although they have achieved protective effects to some extent, the reagents have not been commercialized, and there is no related clinical application reported.

Bisphosphonates are a class of synthetic pyrophosphonic acid analogues ${ }^{3,4}$. The osteoclasts (macrophages settled in the bone) in bone phagocytize the bisphosphonate combined with hydroxyapatite crystal and form ATP analogs with toxic effects in osteoclasts, resulting in cellular energy dysmetabolism that causes the apoptosis of osteoclast and thereby reduces the bone resorption of osteoclasts. Bisphosphonates can selectively eliminate monocytes/macrophages. In this regard, Fukushima et al. ${ }^{27}$ used Clodronate liposome to inhibit the function of macrophages and significantly alleviate the blepharoconjunctivitis caused by immune reaction. Shifrin et al. ${ }^{28}$ used Clodronate liposome to induce macrophage apoptosis in mice, which significantly reduced the local and systemic inflammatory response in severe acute pancreatitis, but has no effect on other cells. Recent studies have shown that as a specific scavenger of macrophages, Clodronate has no effect on other cells such as vascular endothelial cells and smooth muscle cells. ZOD is a bisphosphonate containing nitrogen with greater effect on inducing osteoclast apoptosis and better safety, which has just entered the Chinese market ${ }^{29}$. Zoledronate is superior to disodium chlorondronate on the induction of KC apoptosis in terms of dose- and time- effect relationship. Due to the smaller molecular weight, Zoledronate liposome has better entrapment rate ${ }^{30}$.

Liposome, one of the most common galenic pharmacy methods to reduce drug toxicity, is an ideal drug carrier. The conventional liposomes prepared with lecithin or cholesterol are phagocytized by reticuloendothelial system in vivo and act on the liver and spleen through blood circulation ${ }^{31}$. It has been widely researched experimentally and clinically and its application has been widely used in many fields, especially in medical engineering. As a drug carrier, liposome has the characteristics of improving drug efficacy, reducing adverse drug reactions and targeting ${ }^{32}$.

In this study, a preparation method of Zoledronate liposome was established and its inductive effect on the apoptosis of Kupffer cells in rat liver was observed. There has been animal experiment showed that Zoledronate liposomes would help ZOD to concentrate on target zone, so the concentration in other sites decreased and the toxicity was less ${ }^{33}$.

The results showed that the entrapment rate (\%) of Zoledronate was 43.4 77.8. Immunohistochemistry revealed that the number of Kupffer cells in the liver was 19.3 \pm 2.1 in PBS group and $5.5 \pm 1.7$ in Zoledronate liposome group, with a significant difference $(P<0.05)$. The apoptosis rate of KCs was determined by flow cytometry. It was $4.1 \pm 0.8 \%$ in PBS group and $9 \pm 2.2 \%$ and $23.3 \pm 5.9 \%$ in Zoledronate liposomes with 
different concentrations, showing a significant difference $(P<0.05)$. The results showed that Zoledronate liposomes can effectively induce apoptosis of Kupffer cells in vitro and in vivo, and the apoptosis rate of $\mathrm{KCS}$ is related to the concentration of Zoledronate liposomes. To establish a rat liver Kupffer cell apoptosis model provides a new way to further study the function of Kupffer cells.

\section{References}

1. Pattni BS, Chupin VV, Torchilin VP. New Developments in liposomal drug delivery. Chem Rev. 2015;115(19):10938-66. doi: 10.1021/acs.chemrev.5b00046.

2. Allen TM, Cullis PR. Liposomal drug delivery systems: from concept to clinicalapplications. Adv Drug Deliv Rev. 2013;65(1):36-48. doi: 10.1016/j.addr.2012.09.037.

3. Frith JC, Monkkonen J, Blackburn GM, Russell RG, Rogers MJ. Clodronate and liposomeencapsulated clodronate are metabolized to a toxic ATP analog, adenosine 5'-(beta, gamma-dichloromethylene) triphosphate, by mammalian cells in vitro. J Bone Miner Res. 1997;12(9):1358-67. doi: 10.1359/ jbmr.1997.12.9.1358.

4. Naito $M$, Nagai $H$, Kawano $S$, Umezu $H$, Zhu $\mathrm{H}$, Moriyama $\mathrm{H}$, Yamamoto $\mathrm{T}$, Takatsuka $\mathrm{H}$, Takei Y. Liposome-encapsulated dichloromethylene diphosphonate induces macrophage apoptosis in vivo and in vitro. J Leukoc Biol. 1996;60(3):337-44. doi: 10.1002/jlb.60.3.337.

5. Singh T, Kaur V, Kumar M, Kaur P, Murthy RS, Rawal RK. The critical role of bisphosphonates to target bone cancer metastasis: an overview. J Drug Target. 2015;23(1):1-15. doi: 10.3109/1061186X.2014.950668.

6. Nishisho T, Hanaoka N, Miyagi R, Sakai T, Toki S, Takahashi M, Kenji E, Yasui N, Sairyo K. Local administration of zoledronic acid for giant cell tumor of bone. Orthopedics. 2015;38(1):e25-30. doi: 10.3928/0147744720150105-56.

7. Sanfilippo KM, Gage B, Luo S, Weilbaecher $\mathrm{K}$, Tomasson M, Vij R, Colditz G, Carson K. Comparative effectiveness on survival of zoledronic acid versus pamidronate in multiple myeloma. Leuk
Lymphoma. 2015;56(3):615-21. doi: 10.3109/10428194.2014.924117.

8. Cohen PR. Zoledronic acid-associated symmetrical drug-related intertriginous and flexural exanthema (SDRIFE): report of baboon syndrome in a woman with recurrent metastatic breast cancer after receiving zoledronic acid. Dermatol Online J. 2015;21(8). PMID: 26437156.

9. Honda $Y$, Takahashi S, Zhang $Y$, Ono A, Murakami E, Shi N, Kawaoka T, Miki D, Tsuge M, Hiraga N, Abe H, Ochi H, Imamura M, Aikata $\mathrm{H}$, Chayama K. Effects of bisphosphonate zoledronic acid in hepatocellular carcinoma, depending on mevalonate pathway. J Gastroenterol Hepatol. 2015;30(3):619-27. doi: 10.1111/jgh.12715.

10.Salzano G, Marra M, Porru M, Zappavigna S, Abbruzzese A, La Rotonda MI, Leonetti C, Caraglia M, De Rosa G. Self-assembly nanoparticles for the delivery of bisphosphonates into tumors. Int J Pharm. 2011;403(1-2):292-7. doi: 10.1016/j. ijpharm.2010.10.046.

11. Hattori Y, Shibuya K, Kojima K, Miatmoko A, Kawano K, Ozaki K, Yonemochi E. Zoledronic acid enhances antitumor efficacy of liposomal doxorubicin. Int J Oncol. 2015;47(1):211-9. doi: 10.3892/ ijo.2015.2991.

12.van Rooijen $\mathrm{N}$, van Kesteren-Hendrikx $\mathrm{E}$. "In vivo" depletion of macrophages by liposome-mediated "suicide". Methods Enzymol. 2003;373:3-16. PMID: 14714393.

13.Bilzer M, Roggel F, Gerbes AL. Role of Kupffer cells in host defense and liver disease. Liver Int. 2006;26(10):1175-86. doi: 10.1111/j.1478-3231.2006.01342.x.

14.Otogawa K, Kinoshita K, Fujii H, Sakabe M, Shiga R, Nakatani K, Ikeda K, Nakajima Y, Ikura Y, Ueda M, Arakawa T, Hato F, Kawada N. Erythrophagocytosis by liver macrophages (Kupffer cells) promotes oxidative stress, inflammation, and fibrosis in a rabbit model of steatohepatitis: implications for the pathogenesis of human nonalcoholic steatohepatitis. Am J Pathol. 2007;170(3):967-80. doi: 10.2353/ ajpath.2007.060441.

15.Canbay A, Feldstein AE, Higuchi $H$, Werneburg N, Grambihler A, Bronk SF, Gores GJ. Kupffer cell engulfment of apoptotic bodies stimulates death ligand and cytokine 
expression. Hepatology. 2003;38(5):118898. doi: 10.1053/jhep.2003.50472.

16.Bottcher JP, Knolle PA, Stabenow D. Mechanisms balancing tolerance and immunity in the liver. Dig Dis. 2011;29(4):38490. doi: 10.1159/000329801.

17.Monnier J, Piquet-Pellorce C, Feige JJ, Musso $\mathrm{O}$, Clement $\mathrm{B}$, Turlin $\mathrm{B}$, Theret $\mathrm{N}$, Samson M. Prokineticin 2/Bv8 is expressed in kupffer cells in liver and is down regulated in human hepatocellular carcinoma. World J Gastroenterol. 2008;14(8):1182-91. doi: 10.3748/wjg.14.1182.

18. Liu G, Ma H, Jiang L, Zhao Y. Allograft inflammatory factor-1 and its immune regulation. Autoimmunity. 2007;40(2):95102. doi: $10.1080 / 08916930601083946$.

19.Chen Y, Liu Z, Liang S, Luan X, Long F, Chen J, Peng Y, Yan L, Gong J. Role of kupffer cells in the induction of tolerance of orthotopic liver transplantation in rats. Liver Transpl. 2008;14(6):823-36. doi: 10.1002/lt.21450.

20.Briceño J, Ciria R. Early graft dysfunction after liver transplantation. Transplant Proc. 2010;42(2):631-3. doi: 10.1016/j. transproceed.2010.02.004.

21. Hanschen $M$, Zahler S, Krombach $F$, Khandoga A. Reciprocal activation between $\mathrm{CD}^{+} \mathrm{T}$ cells and kupffer cells during hepatic ischemia-reperfusion. Transplantation. 2008;86(5):710-8. doi: 10.1097/ TP.0b013e3181821aa7.

22.Montalvo-Jave EE, Escalante-Tattersfield T, Ortega-Salgado JA, Piña E, Geller DA. Factors in the pathophysiology of the liver ischemia-reperfusion injury. J Surg Res. 2008;147(1):153-9. doi: 10.1016/j. jss.2007.06.015.

23.Kincius $M$, Liang $R$, Nickkholgh $A$, Hoffmann $\mathrm{K}$, Flechtenmacher C, Ryschich E, Gutt CN, Gebhard MM, Schmidt J, Büchler MW, Schemmer P. Taurine protects from liver injury after warm ischemia in rats: the role of kupffer cells. Eur Surg Res. 2007;39(5):27583. doi: 10.1159/000102982.

24.Tomiyama K, Ikeda A, Ueki S, Nakao A, Stolz DB, Koike Y, Afrazi A, Gandhi C, Tokita D, Geller DA, Murase N. Inhibition of Kupffer cellmediated early proinflammatory response with carbon monoxide in transplant-induced hepatic ischemia/reperfusion injury in rats. Hepatology. 2008;48(5):1608-20. doi: 10.1002/hep.22482.
25.Zeng Z, Huang HF, Chen MQ, Song F, Zhang YJ. Heme oxygenase-1 protects donor livers from ischemia/reperfusion injury: the role of Kupffer cells. World J Gastroenterol. 2010;16(10):1285-92. doi: 10.3748/wjg. v16.i10.1285.

26.Jahnke C, Mehrabi A, Golling M, Frankenberg MV, Kashfi A, Nentwich $H$, Fonouni $H$, Nickkholgh A, Schemmer P, Gutt CN, Weitz J, Schmidt J, Gebhard MM, Büchler MW, Kraus T. Evaluation of microperfusion disturbances in the transplanted liver after Kupffer cell destruction using $\mathrm{GdCl} 3$ : an experimental porcine study. Transplant Proc. 2006;38(5):1588-95. doi: 10.1016/j. transproceed.2006.02.067.

27.Fukushima A, Ozaki A, Ishida W, Rooijen NV, Fukata K, Ueno $H$. Suppression of macrophage infiltration into the conjunctiva by clodronate liposomes in experimental immune-mediated blepharoconjunctivitis. Cell Biol Int. 2005;29(4):277-86. doi: 10.1016/j.cellbi.2004.12.011.

28.Shifrin AL, Chirmule N, Zhang Y, Raper SE. Macrophage ablation attenuates adenoviral vector-induced pancreatitis. Surgery. 2005;137(5):545-51. doi: 10.1016/j. surg.2005.01.004.

29.Li F, Wang W, Li L, Chang Y, Su D, Guo G, He X, Li M. An effective therapy to painful bone metastases: cryoablation combined with zoledronic acid. Pathol Oncol Res. 2014;20(4):885-91. doi: 10.1007/s12253014-9769-7.

30.Zlatev HP, Auriola S, Mönkkönen J, Määttä JA. Uptake of free, calcium-bound and liposomal encapsulated nitrogen containing bisphosphonates by breast cancer cells. Eur J Pharm Sci. 2016;86:58-66. doi: 10.1016/j. ejps.2016.02.016.

31.Caron WP, Lay JC, Fong AM, La-Beck NM, Kumar $\mathrm{P}$, Newman SE, Zhou $\mathrm{H}$, Monaco JH, Clarke-Pearson DL, Brewster WR, Van Le L, Bae-Jump VL, Gehrig PA, Zamboni WC. Translational studies of phenotypic probes for the mononuclear phagocyte system and liposomal pharmacology. J Pharmacol Exp Ther. 2013;347(3):599-606. doi: 10.1124/ jpet.113.208801.

32. Kumar N, Rai A, Reddy ND, Raj PV, Jain P, Deshpande P, Mathew G, Kutty NG, Udupa N, Rao CM. Silymarin liposomes improves oral bioavailability of silybin besides targeting 
hepatocytes, and immune cells. Pharmacol Rep. 2014;66(5):788-98. doi: 10.1016/j. pharep.2014.04.007.

33. Ho EA, Ramsay $E$, Ginj $M$, Anantha $M$, Bregman I, Sy J, Woo J, Osooly-Talesh M, Yapp DT, Bally MB. Characterization of cationic liposome formulations designed to exhibit extended plasma residence times and tumor vasculature targeting properties. J Pharm Sci. 2010;99(6):2839-53. doi: 10.1002/jps.22043.

\section{Correspondence:}

Li-Qun Pang

Department of General Surgery

The Affiliated Huaian No.1 People's Hospital of

Nanjing Medical University

Huai'an 223300 China

Phone: +8613515248309

Fax: +8651783165499

cnliqunpang@163.com

Received: Aug 10, 2018

Review: Oct 13, 2018

Accepted: Nov 16, 2018
Conflict of interest: none

Financial source: Huai'an Science and Technology Bureau International Cooperation Project (HG201112)
${ }^{1}$ Research performed at Central Laboratory, Affiliated Huaian No.1 People's Hospital of Nanjing Medical University, China. 